\title{
Phylogenetic and morphological investigation of a Dunaliella strain isolated from Yuncheng Salt Lake, China
}

\author{
Feipeng Wang, Jia Feng, Shulian Xie* \\ School of Life Science, Shanxi University, Taiyuan 030006, China
}

Email address:

xies1@sxu.edu.cn (Shulian Xie)

To cite this article:

Feipeng Wang, Jia Feng, Shulian Xie. Phylogenetic and Morphological Investigation of a Dunaliella Strain Isolated from Yuncheng Salt Lake, China. Plant. Vol. 2, No. 2, 2014, pp. 20-26. doi: 10.11648/j.plant.20140202.12

\begin{abstract}
A Dunaliella strain was isolated from Yuncheng Salt Lake, Shanxi, China $\left(111.05^{\circ} \mathrm{E}, 35.03^{\circ} \mathrm{N}\right)$. Morphological characteristics and molecular data were used to evaluate the relationship of this algal strain to other Dunaliella strains. Morphology of the isolated strain observed was close to Dunaliella salina. Phylogenetic trees were constructed from $r b c L$, $p s a B$, ITS (ITS-1+5.8S rDNA+ITS-2) and 18S rDNA sequence data. Phylogenetic analysis of the four gene sequences revealed that the isolated Dunaliella strain is closely related to D. salina (Dunal) Teodoresco, D. peircei Nicolai et Baas-Becking, D. tertiolecta Butcher and more likely closer to D. salina. Combined morphological characteristics and phylogenetic analysis, the isolated Duanliella — designated here as D. sp YC01—should be a Dunalialla salina strain.
\end{abstract}

Keywords: Dunaliella, Morphology, Phylogeny

\section{Introduction}

The genus Dunaliella comprises a group of unicellular, biflagellated green algae that lack cell walls. Cells are ovoid, spherical, or ellipsoid [1]. Members of this genus are the only eukaryotic, photosynthetic organisms known to grow over an extremely wide range of salt concentrations, varying from $0.5 \mathrm{M} \mathrm{NaCl}$ up to saturation $(5.5 \mathrm{M})[2,3]$. This salt adaption ability is attributed to the production of intracellular glycerol, which acts as an osmotic compatible solute [4]. Dunaliella may thus be a convenient model organism for studying salt adaptation in algae [5]. Under certain conditions, such as high light intensity exposure and nutrient limitation, some Dunaliella species can accumulate high $\beta$-carotene levels equivalent to $10 \%$ of their dry biomass [6]. $\beta$-carotene is a major source of vitamin A, necessary for proper retinal function, and affects many tissue types [7]. Humans cannot synthesize necessary carotenoids, however, and must obtain them from their diets. Because Dunaliella is an ideal natural source of $\beta$-carotene, Dunaliella $\beta$-carotene production plants are in operation in Asia, North America, and Australia [8].

Since the first formal description of Dunaliella by Teodoresco [9], species of this genus have been described from a wide variety of habitats [10-12]. Based on a species-level analysis of morphological and structural features combined with physiological and biochemical characteristics, Massyuk [13-15] recognized 29 species, and divided the genus into two subgenera, Pascheria and Dunaliella. Members of the genus can adapt to extreme environments. For example, $D$. acidophila is able to grow in highly acidic environments $(\mathrm{pH} 0-1)$, while a novel subaerial species, D. atacamensis, has been described growing on cave spider webs in the Atacama Desert [16]. Because Dunaliella species lack cell walls, their morphologies vary under different growing conditions, making characterization difficult. In spite of the excellent work of Massyuk, several strains in culture collections are misidentified, and some species described as separate entities may actually be polymorphic forms of the same taxon [17]. Recent studies have confirmed that molecular methods are useful tools for distinguishing between Dunaliella taxa with similar morphologies at inter- and intra-specific levels [18-19]. Combination of morphological data with molecular methods should thus provide a better understanding of Dunaliella taxonomy.

In an earlier study, Li and Xie [20] reported the existence of Dunaliella in Yuncheng Salt Lake, Shanxi, China. In the study reported here, we isolated and cultured the strain from Yuncheng Salt Lake, and performed a taxonomic and phylogenetic analysis of this micro-alga using both morphological and molecular methods. 


\section{Material and Methods}

\subsection{Sample Collection and Isolation}

Samples were collected from Yuncheng Salt Lake, Shanxi, China $\left(111.05^{\circ} \mathrm{E}, 35.03^{\circ} \mathrm{N}\right.$; Fig. 1). Lake water $\mathrm{pH}$ was $7.2-7.6$, and its salinity was above $0.1 \mathrm{~g} \mathrm{ml}^{-1}$. Isolation and purification of Dunaliella cells was performed according to previously described methods [21]. The isolated Dunaliella strain was designated as $D$. sp. YC01 in this study.

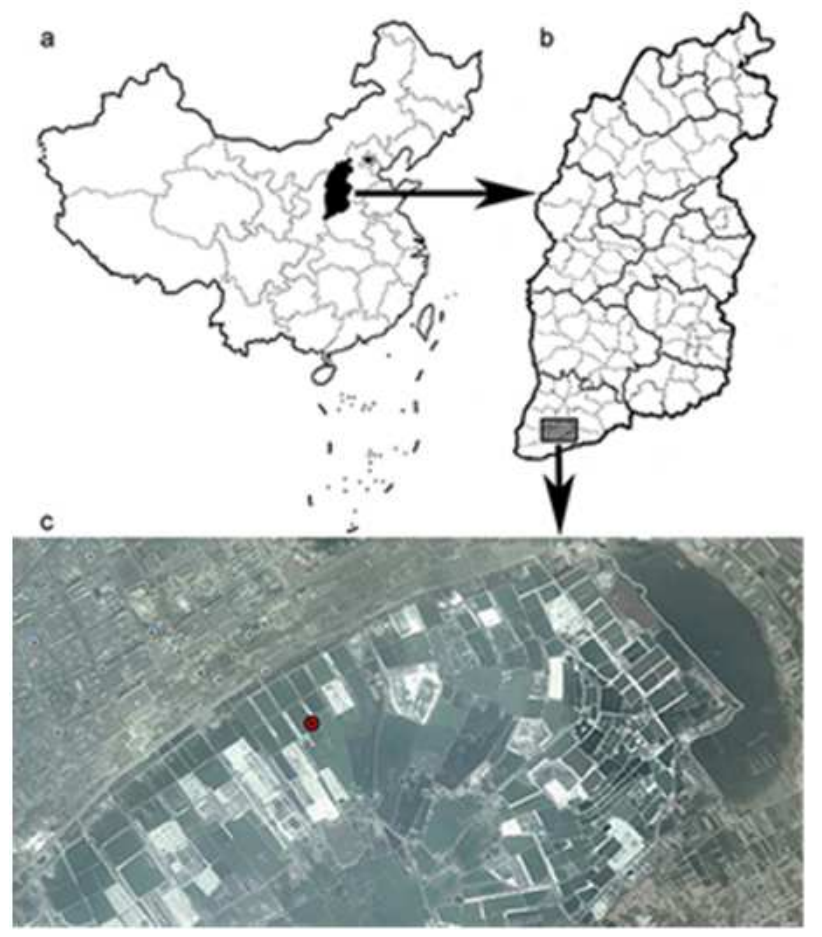

a. The map of China. The shaded part is Shanxi; b. The map of Shanxi. The shaded part is the location of Yuncheng Salt Lake; c. The map of Yuncheng Salt Lake. The marked part is the sample collection location.

Figure 1. Sample collection location

\subsection{Culture Conditions}

The isolated and purified alga was cultured on medium containing $2.05 \mathrm{M} \mathrm{NaCl} 17.64 \mathrm{mM}, \mathrm{NaNO}_{3}, 0.22 \mathrm{mM}$ $\mathrm{K}_{2} \mathrm{HPO}_{4}, 0.3 \mathrm{mM} \mathrm{MgSO} \cdot \cdot 7 \mathrm{H}_{2} \mathrm{O}, 0.32 \mathrm{mM} \mathrm{CaCl} \cdot 2 \mathrm{HO}_{2}$, $0.18 \quad \mathrm{mM} \quad \mathrm{Na}_{2} \mathrm{CO}_{3}, \quad 0.02 \quad \mathrm{mM} \quad \mathrm{C}_{6} \mathrm{H}_{8} \mathrm{O}_{7} \cdot \mathrm{H}_{2} \mathrm{O}, \quad 10 \mu \mathrm{M}$ $\mathrm{Fe}\left(\mathrm{NH}_{4}\right)_{3}\left(\mathrm{C}_{6} \mathrm{H}_{2} \mathrm{O}_{7}\right), 46.1 \mu \mathrm{M} \mathrm{H}_{3} \mathrm{BO}_{3}, 9.39 \mu \mathrm{M} \mathrm{MnCl}_{2} \cdot 4 \mathrm{H}_{2} \mathrm{O}$, $0.77 \mu \mathrm{M} \mathrm{ZnSO} \cdot \cdot 7 \mathrm{H}_{2} \mathrm{O}, 1.61 \mu \mathrm{M} \mathrm{Na} 2 \mathrm{MoO}_{4} \cdot 2 \mathrm{H}_{2} \mathrm{O}, 0.32 \mu \mathrm{M}$ $\mathrm{CuSO}_{4} \cdot 5 \mathrm{H}_{2} \mathrm{O}$, and $0.172 \mu \mathrm{M} \mathrm{Co}\left(\mathrm{NO}_{3}\right) \cdot 6 \mathrm{H}_{2} \mathrm{O}$. Dunaliella sp. YC01 was grown in a 250-ml Erlenmeyer flask containing $100 \mathrm{ml}$ medium at $25 \pm 1^{\circ} \mathrm{C}$, which was manually shaken twice daily and subjected to a $12 \mathrm{~h}: 12 \mathrm{~h}$ light/dark photoperiod provided by cool white fluorescent lamps.

\subsection{DNA Methods}

After approximately 3 weeks, cultured cells were harvested by centrifugation for $10 \mathrm{~min}$ at $5000 \mathrm{xg}$. Genomic DNA was extracted using the CTAB protocol [22]. For amplification of the nuclear $18 \mathrm{~S}$ rDNA gene region,
Dunaliella-specific primers MA1 (5'-CGGGATCCGTAGTCATATGCTTGTCTC-3') and MA2 (5'-CGGAATTCCTTCTGCAGGTTCACC-3') was used [23]. The chloroplast $r b c L$ gene region was amplified using primers 475-497 (5'-CGTGACAAACTAAACAAATATGG-3') and 1181-1160 (5'-AAGATTTCAACTAAAGCTGGCA-3'). The nuclear rDNA ITS region (ITS-1+5.8S rDNA+ITS-2) was amplified using universal primers AB28 (5'-GGGATCCATATGCTTAAGTTCAGCGGGT-3') and TW81 (5'-GGGATCCGTTTCCGTAGGTGAACCTGC-3'). The chloroplast $p s a B$ gene was amplified using the primers DunpsaBFw22 (5'-ATTTGGGATCCACATTTTGGT-3') and DunapsaBRv753 (5'-TACTGAAGCTAAAGCTAAA-3') [16]. Polymerase chain reactions (PCRs) were performed in a MyCycler thermal cycler (Bio-Rad, California, USA). PCR amplifications of $18 \mathrm{~S}$ rDNA, $p s a B$, and $r b c L$ regions were carried out as follows: initial denaturation at $94^{\circ} \mathrm{C}$ for 5 min, followed either by 30 cycles of $94^{\circ} \mathrm{C}$ for $1 \mathrm{~min}, 58^{\circ} \mathrm{C}$ for $1 \mathrm{~min}$, and $72^{\circ} \mathrm{C}$ for $2 \mathrm{~min}$ for $18 \mathrm{~S}$ rDNA, 30 cycles of $93^{\circ} \mathrm{C}$ for $1 \mathrm{~min}, 52^{\circ} \mathrm{C}$ for $1 \mathrm{~min}$, and $72^{\circ} \mathrm{C}$ for $1 \mathrm{~min}$ for $p s a B$, or 30 cycles of $94^{\circ} \mathrm{C}$ for $1 \mathrm{~min}, 62^{\circ} \mathrm{C}$ for $50 \mathrm{~s}$, and $72^{\circ} \mathrm{C}$ for 1 $\min$ for $r b c L$, with a final extension of $8 \mathrm{~min}$ at $72^{\circ} \mathrm{C}$. For the ITS (ITS-1+5.8S rDNA+ITS-2) region, PCRs were performed as described previously [19]. PCR products were analyzed by $1 \%$ agarose gel electrophoresis and purified using a Sangon agarose gel DNA extraction kit. The purified products were sent to Sangon Biotech (Shanghai, China) for sequencing.

\subsection{Phylogenetic Analyses}

Phylogenetic analyses were performed using $18 \mathrm{~S}$ rDNA, $r b c L$, psaB, and ITS sequence data. Multiple alignments incorporating sequences of other Dunaliella strains obtained from the National Center for Biotechnology Information (NCBI) nucleotide database (Table 1) were performed using CLUSTALW [24], with manual adjustments as needed. Phylogenetic trees were constructed from the aligned gene sequences using neighbor-joining (NJ), maximum likelihood (ML), and Bayesian (BI) methods. NJ, ML, and BI analyses were performed using MEGA5 [25], PhyML 3.0 [26], and MrBayes version 3.1.2 [27], respectively. For NJ analyses, evolutionary distances were computed using the Kimura 2-parameter method with 1000 bootstrap replicates. The program jModeltest [28] was used to determine the best-fitting models of sequence evolution for ML and BI methods. The model selected for the $18 \mathrm{~S}$ rDNA data was $\operatorname{TrN}+\mathrm{I}+\Gamma$, with the following parameters used: invariant sites $=0.8532$, gamma distribution $=0.6780$; base frequencies $\mathrm{A}$ $=0.2494, \mathrm{C}=0.2100, \mathrm{G}=0.2755, \mathrm{~T}=0.2651$; rate matrix $\mathrm{A}-\mathrm{C}=\mathrm{A}-\mathrm{T}=\mathrm{C}-\mathrm{G}=\mathrm{G}-\mathrm{T}=1, \mathrm{~A}-\mathrm{G}=4.5699, \mathrm{C}-\mathrm{T}=11.4595$. For the $r b c L$ gene, we used a TPM 3 uf $+\mathrm{I}+\Gamma$ model, with the following parameters: invariant sites $=0.5750$, gamma distribution $=0.5830$; base frequencies $\mathrm{A}=0.2879, \mathrm{C}=$ $0.2084, \mathrm{G}=0.1878, \mathrm{~T}=0.3159$; rate matrix $\mathrm{A}-\mathrm{C}=\mathrm{C}-\mathrm{G}=$ $0.0970, \mathrm{~A}-\mathrm{G}=\mathrm{C}-\mathrm{T}=1.1090, \mathrm{~A}-\mathrm{T}=\mathrm{C}-\mathrm{T}=1$. Analysis of the $p s a B$ gene data was carried out under a $\mathrm{GTR}+\Gamma$ model, with gamma distribution $=0.2230$ and base frequencies $\mathrm{A}=$ 
$0.2417, \mathrm{C}=0.1841, \mathrm{G}=0.1850$, and $\mathrm{T}=03892$. The model selected for the ITS region was $\operatorname{TrNef}+\Gamma$, with gamma distribution $=0.4337$, rate matrix A-C $=\mathrm{A}-\mathrm{T}=\mathrm{C}-\mathrm{G}=\mathrm{G}-\mathrm{T}=$ $1, \mathrm{~A}-\mathrm{G}=2.5767$, and $\mathrm{C}-\mathrm{T}=7.0137 . \mathrm{D}$. sp. $\mathrm{YC} 0118 \mathrm{~S}$ rDNA, $r b c L$, ITS, and $p s a B$ sequences were deposited in GenBank with accession numbers KF054056, KF054057, KF054058, and KF054059, respectively.

\section{Results}

\subsection{Morphological Observations}

Dunaliella sp. YC01 cells were examined and photographed under an optical microscope (Olympus BX-51, Japan). The cells were varied in shape from pyriform to round or ovoid. The cells contained one cup-shaped chloroplast occupying about half of the cell interior, and a single pyrenoid surrounded by several starch bodies. At the flagellar end of cells, a papilla was usually observed in young cells. Flagella were a bit longer than the length of the cell (Fig. 2).

\subsection{Phylogenetic Analyses}

PCR amplification of $r b c L, p s a B$, ITS, and 18S rDNA regions of Dunaliella sp. YC01 produced 731-, 1,197-, 727-, and 1,721-bp amplicons, respectively. ML, NJ, and BI trees based on each DNA region were constructed from individual aligned datasets comprising sequences of $D$. sp. YC01and other Dunaliella strains. For the four genes, topologies recovered based on the BI algorithm are shown in Figs 3-6, respectively, with ML and NJ bootstrap support values indicated.

In the phylogenetic tree generated by BI analysis of $r b c L$ sequences (Fig. 3), D. sp. YC01 is grouped together with $D$. peircei Nicolai et Baas-Becking strain UTEX 2192 (DQ313196) and D. salina (Dunal) Teodoresco strain UTEX 200 (DQ313197) in a well-supported clade (ML bootstrap value/NJ bootstrap value/BI posterior probability of $99.3 \% / 100 \% / 1.00)$. D. sp. YC01 is also shown to be closely related to $D$. sp. MBTD-CMFRI-S086 (JN797810), which is sister to the above clade with ML bootstrap values and BI posterior probabilities of $45.6 \%$ and 0.91 , respectively. The ML tree was rooted using a published $r b c L$ sequence of Chlamydomonas reinhardtii P. A. Dangeard as an outgroup [29].

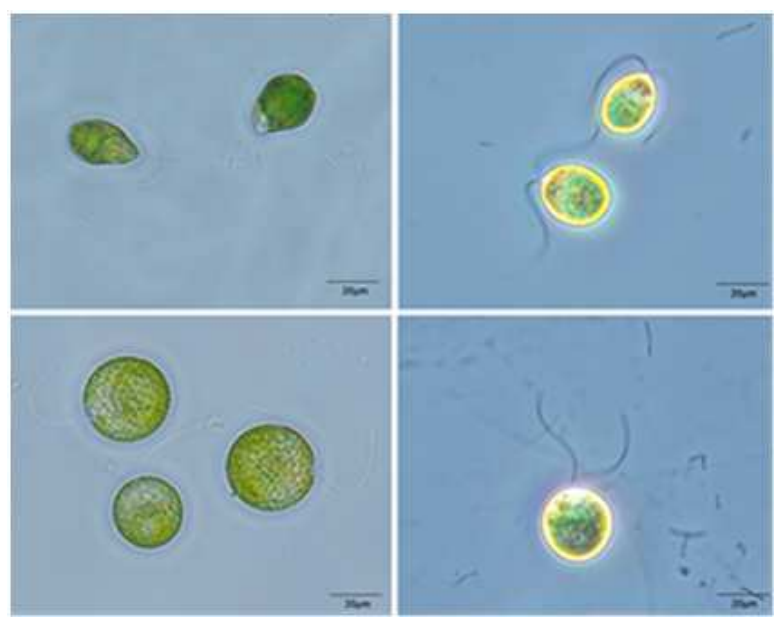

Figure 2. Morphology of Dunaliella sp. YCO1

In the $\mathrm{BI}$ tree obtained using aligned $p s a B$ gene sequences (Fig. 4), D. sp. YC01 is sister to D. tertiolecta Butcher (JQ039051). These two strains are placed into a strongly-supported clade $(\mathrm{ML} / \mathrm{NJ} / \mathrm{BI}$ support = $100 \% / 100 \% / 1.00)$ along with $D$. parva W.Lerche strain UTEX 1983 (AB084375) and D. salina (AY820754). The four Dunaliella species constitute a monophyletic group in the phylogenetic tree. The tree was rooted using Chloromonas radiata (T.R.Deason et H.C.Bold ) T.PrÖschold, B.Marin, U.W.SchlÖsser et M.Melkonian [16] as an outgroup.

Using the universal primers AB28 and TW81, the nuclear rDNA ITS region (ITS-1+5.8 rDNA+ITS-2) of $D$. sp. YC01 was amplified; its sequence length, $727 \mathrm{bp}$, was similar to that of other Dunaliella strains in the NCBI database. In the BI tree obtained using aligned ITS gene sequences (Fig. 5). Dunaliella sp. YC01 is sister to D. salina strain 9802 (EF695045) in a well-supported clade (ML bootstrap value/NJ bootstrap value/BI posterior probability of 98.4\%/94\%/1.00). This tree was rooted using Chlamydomonas reinhardtii [29] as an outgroup.

BI analysis of nuclear $18 \mathrm{~S}$ rDNA data recovered a phylogenetic tree (Fig. 6) in which $D$. sp. YC01 is sister to $D$. salina (AF506698). These two strains constitute a clade $(\mathrm{ML} / \mathrm{BI}$ support $=83.4 \% / 0.99)$ together with $D$. salina strain UTEX LB 1644 (DQ009765). The phylogenetic tree was rooted using a published sequence of Asteromonas gracilis Artari strain CCMP 813 [16] as an outgroup. Asteromonas is a genus in Asteromonadaceae, the sister family in the Chlamydomonadales to Dunaliellaceae [30-31]. 


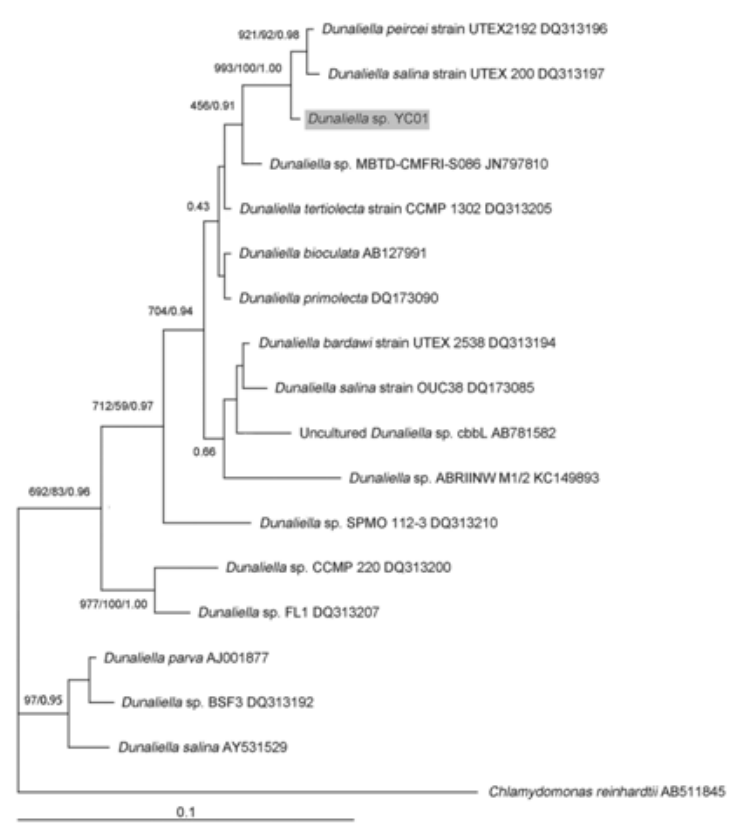

Figure 3. Phylogenetic tree derived from Bayesian (BI) analysis of aligned $r b c L$ gene sequences of Dunaliella species. Numbers at nodes indicate $M L$ and neighbor-joining (NJ) bootstrap support values derived from 1000 bootstrap replicates, and Bayesian (BI) posterior probabilities (ML/NJ/BI)

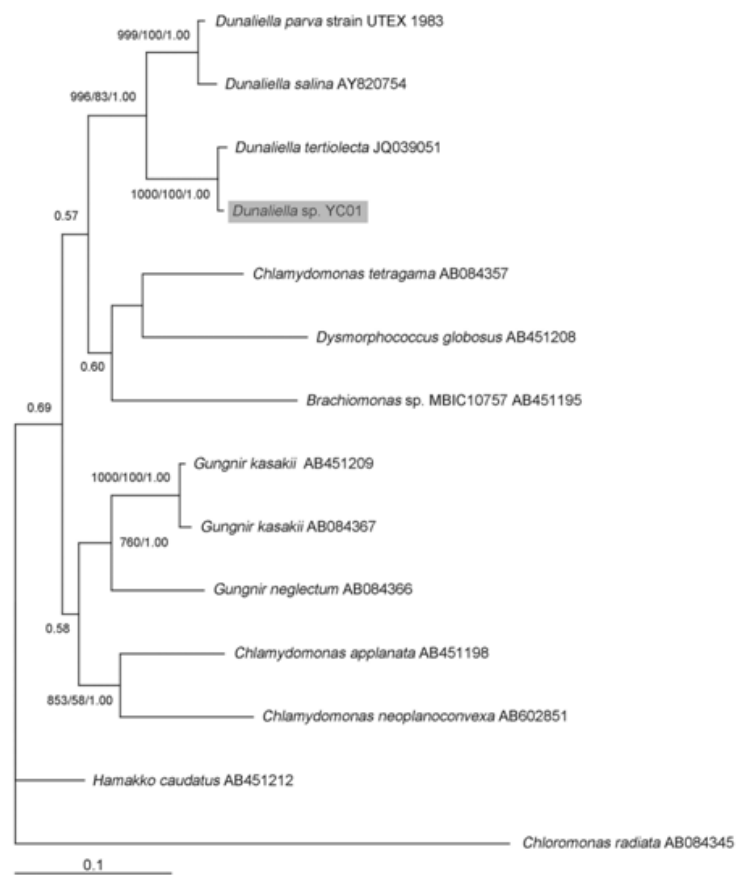

Figure 4. Phylogenetic tree derived from Bayesian (BI) analysis of aligned psaB gene sequences. Numbers at nodes indicate ML and neighbor-joining (NJ) bootstrap support values derived from 1000 bootstrap replicates, and Bayesian (BI) posterior probabilities (ML/NJ/BI)

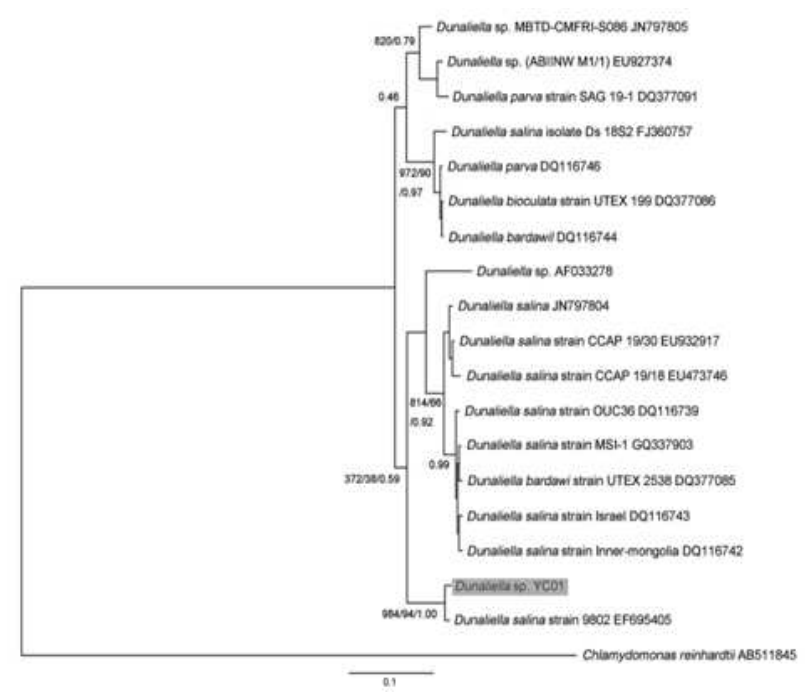

Figure 5. Phylogenetic tree derived from Bayesian (BI) analysis of aligned ITS sequences of Dunaliella species. Numbers at nodes indicate maximum likelihood (ML) and NJ bootstrap support values derived from 1000 bootstrap replicates, and Bayesian (BI) posterior probabilities (ML/NJ/BI)

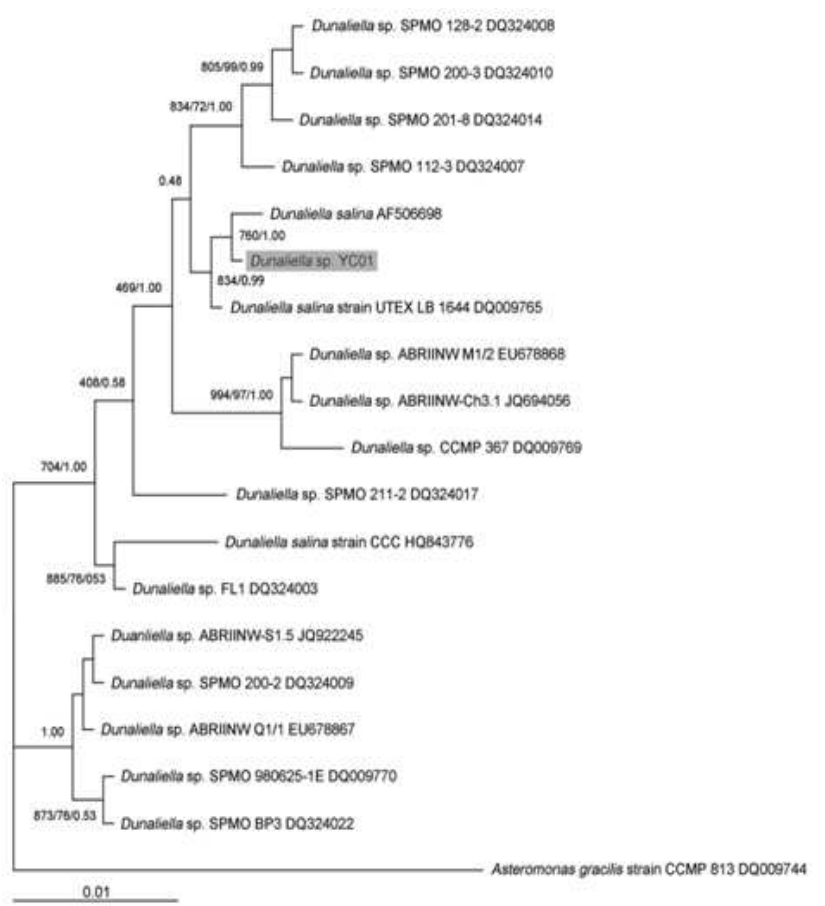

Figure 6. Phylogenetic tree derived from Bayesian (BI) analysis of aligned 18S rDNA gene sequences of Dunaliella species. Numbers at nodes indicate maximum likelihood (ML) and NJ bootstrap support values derived from 1000 bootstrap replicates, and Bayesian (BI) posterior probabilities $(M L / N J / B I)$

Table 1. Strains of Dunaliella analyzed in this study, with their geographic origins and GenBank accession numbers

\begin{tabular}{|c|c|c|c|c|}
\hline Dunaliella strains & $18 S$ rDNA & psaB & ITS & Geographic Origin \\
\hline Dunaliella bardawil & & & DQ116744 & \\
\hline Dunaliella bardawi (UTEX 2538) & & DQ313194 & DQ377085 & $\begin{array}{l}\text { salt pond nearBardawil lagoon, North } \\
\text { Sinai, } \\
\text { Israel }\end{array}$ \\
\hline Dunaliella bioculata & & AB127991 & & \\
\hline Dunaliella bioculata (UTEX 199) & & & DQ377086 & Soviet Union \\
\hline
\end{tabular}




\begin{tabular}{|c|c|c|c|c|c|}
\hline Dunaliella strains & 18S rDNA & $r b c L$ & psaB & ITS & Geographic Origin \\
\hline Dunaliella parva & & AJ001877 & & & \\
\hline Dunaliella parva & & & & DQ116746 & \\
\hline Dunaliella parva (UTEX 1983) & & & AB084375 & & \\
\hline Dunaliella parva (SAG 19-1) & & & & DQ377091 & LaculSarat, Romania \\
\hline Dunaliella peircei (UTEX2192) & & DQ313196 & & & Salt flat \\
\hline Dunaliella primolecta & & DQ173090 & & & \\
\hline Dunaliella salina & AF506698 & & & & \\
\hline Dunaliella salina & & AY531529 & AY820754 & & \\
\hline Dunaliella salina & & & & JN797804 & Indian \\
\hline Dunaliella salina $(9802)$ & & & & EF695405 & \\
\hline Dunaliella salina (CCAP 19/18) & & & & EF473746 & Hutt Lagoon, Western Australia \\
\hline Dunaliella salina (CCAP 19/30) & & & & EU932917 & $\begin{array}{l}\text { salt pond nearBardawil lagoon, North } \\
\text { Sinai,Israel }\end{array}$ \\
\hline Dunaliella salina $(\mathrm{CCC})$ & HQ843776 & & & & Sambar lake, India \\
\hline Dunaliella salina (Ds 18S2) & & & & FJ360757 & \\
\hline Dunaliella salina (Inner-mongolia) & & & & DQ116742 & Inner-Mongolia \\
\hline Dunaliella salina (Israel) & & & & DQ116743 & Israel \\
\hline Dunaliella salina (MSI-1) & & & & GQ337903 & Maharlu salt lake, Shiraz \\
\hline Dunaliella salina (OUC36) & & & & DQ116739 & \\
\hline Dunaliella salina (OUC38) & & DQ173085 & & & \\
\hline Dunaliella salina (UTEX 200) & & DQ313197 & & & Salt flat \\
\hline Dunaliella salina (UTEX LB 1644) & DQ009765 & & & & Baja, Califomia, USA \\
\hline Dunaliella sp. & & & & AF033278 & \\
\hline Dunaliella sp. (ABRIINW-Ch3.1) & JQ694056 & & & & Iran \\
\hline Dunaliella sp. (ABIINW M1/1) & & & & EU927374 & Iran \\
\hline Dunaliella sp. (ABRIINW M1/2) & EU678868 & KC149893 & & & Iran \\
\hline Dunaliella sp. (ABRIINW Q1/1) & EU678867 & & & & Iran \\
\hline Duanliella sp. (ABRIINW-S1.5) & JQ922245 & & & & Iran \\
\hline Dunaliella sp. (BSF3) & & DQ313192 & & & Salt flat \\
\hline Dunaliella sp. (CCMP 220) & & DQ313200 & & & \\
\hline Dunaliella sp. (CCMP 367) & DQ009769 & & & & \\
\hline Dunaliella sp.(FL1) & DQ324003 & DQ313207 & & & Salt flat \\
\hline Dunaliella sp. (MBTD-CMFRI-S086) & & JN797810 & & JN797805 & Indian \\
\hline Dunaliella sp. (SPMO 112-3) & DQ324007 & DQ313210 & & & Salt flat \\
\hline Dunaliella sp. (SPMO 128-2) & DQ324008 & & & & Salt flat \\
\hline Dunaliella sp. (SPMO 200-2) & DQ324009 & & & & Salt flat \\
\hline Dunaliella sp. (SPMO 200-3) & DQ324010 & & & & Salt flat \\
\hline Dunaliella sp. (SPMO 201-8) & DQ324014 & & & & Salt flat \\
\hline Dunaliella sp. (SPMO 211-2) & DQ324017 & & & & Salt flat \\
\hline Dunaliella sp. (SPMO BP3) & DQ324022 & & & & Salt flat \\
\hline Dunaliella sp. (SPMO 980625-1E) & DQ009770 & & & & Salt flat \\
\hline Dunaliella tertiolecta & & & JQ039051 & & \\
\hline Dunaliella tertiolecta (CCMP 1302) & & DQ313205 & & & \\
\hline Uncultured Dunaliella sp. (cbbL) & & AB781582 & & & Egyptian \\
\hline
\end{tabular}

\section{Discussion}

Morphology of Dunaliella sp. YC01 observed was close to Dunaliella salina, and with limit of nutrient, the green $D$. sp. YC01 in medium would turn to red, which is consistent to D. salina.

Several different molecular marks, primarily $18 \mathrm{~S}$ rDNA, $r b c L$, ITS, and $p s a B$, have previously been used for phylogenetic analysis of Dunaliella [23, 16, 3, 19, 29]. Because these molecular marks have different evolutionary rates, they can be used for inter- and intra-specific classification of Dunaliella. In our study, phylogenetic analysis of the $r b c L$ gene indicated that the closest relatives of D. sp.YC01 are D. peircei strain UTEX 2192 (DQ313196) and D. salina strain UTEX 200 (DQ313197). Blast searching against psaB gene sequences in GenBank uncovered only three Dunaliella species with high identity to $D$. sp. YC01. Our phylogenetic analysis based on this gene indicated that D. tertiolecta (JQ039051) is closely related to $D$. sp. YC01. Phylogenetic analysis of $18 \mathrm{~S}$ rDNA indicated that the closest relatives of $D$. sp. YC01 are $D$. salina (AF506698) and D. salina strain UTEX LB 1644 (DQ009765). Phylogenetic analysis of ITS gene indicated that the closest relative of $D$. sp. YC01 is $D$. salina strain 9802 (EF695405).

Phylogenetic analysis with the four molecular makers indicated the relatives of $D$. sp YC01 are D. salina, $D$. peircei and $D$. tertiolecta, and $D$. salina is more likely closer to $D$. sp YC01.

\section{Conclusion}

Since morphological characteristics of Dunaliella sp. YC01 is similar to D. salina, and phylogenetic analysis with $r b c L, p s a B$, ITS and 18S rDNA gene sequences indicated 
that the most likely relative of $D$. sp YC01 is D. salina. Combined morphological characteristics and phylogenetic analysis, $D$. sp. YC01 should be a $D$. salina strain.

\section{References}

[1] Ben-Amotz A. 1980. Glycerol production in the alga Dunaliella. In: Biochemical and Photosynthetic Aspects of Energy Production (Ed.). A. San Pietro. Academic Press, New York, pp. 191-208.

[2] Liska A. J., Shevchenko A., Pick U. and Katz A. 2004. Enhanced photosynthesis and redox energy production contribute to salinity tolerance in Dunaliella as revealed by homology-base proteomics. Plant Physiol. 136 (1): 2806-2817.

[3] Kannan P. R., Divya S., Rengasamy R., Jayappriyan, K. R. and Rajkumar, R. 2010. Significance of 18S rDNA specific primers in the identification of genus Dunaliella. J. Exp. Sci. $1(1): 27-31$.

[4] Chen H. and Jiang J. G. 2009. Osmotic responses of Dunaliella to the changes of salinity. J. Cell. Physiol. 219 (2): 251-258.

[5] Oren A. 2005. A hundred years of Dunaliella research: 1905-2005. Saline Systems. 1 (2): 1-14.

[6] Ben-Amotz A., Katz A. and Avron M. 1982. Accumulation of $\beta$-carotene in halotolerant algae: purification and characterization of $\beta$-carotene-rich globules from Dunaliella bardawil. J. Phycol. 18: 529-537.

[7] von Lintig J., Hessel S., Isken A., Kiefer C., Lampert J. M., Voolstra O. and Vogt K. 2005. Towards a better understanding of carotenoid metabolism in animals. Bba-Mol. Basis. Dis. 1740 (2): 122-131.

[8] Mercedes, Moreno J., Manzano J. C., Florencio J. and Guerrero M. 2005. Production of Dunaliella salina biomass rich in9-cis-@b-carotene and lutein in a closed tubular photobioreactor. J. Biotechnol. $115 \quad$ (1): 10 , http://dx.doi.org/10.1016/j.jbiotec.2004.07.010.

[9] Teodores E. C. 1905. Organisation et développement du Dunaliella, nouveau genre de Volvocacée-Polyblépharidé. Beih. z. Bot. Zentr. 18 (Abt. 1): 215-232.

[10] Lerche W. 1937. Untersuchungen über Entwicklung und Fortpflanzung in der Gattung Dunaliella. Archiv. f. Protist. 88: 236-269.

[11] Buther R. W. 1959a. An introductory account of the smaller algae of British coastal waters. Part I. Introduction and Chorophyceae. Ministry of Agriculture, Fisheries and Food, Great Britain, Fisheries. Investigations Series IV, pp, 1-74.

[12] Buther R.W. 1959b. An introductory account of the smaller algae of British coastal waters. Hydrobiologia. 12: 249-250.

[13] Msassyuk N. P. 1973a. New taxa of the genus Dunaliella Teod. I. Ukr. Bot. Zh., pp. 30: 175.

[14] Msassyuk N. P. 1973b. New taxa of the genus Dunaliella Teod. II. Ukr. Bot. Zh., pp. 30: 345.

[15] Msassyuk N. P. 1973c. Morphology, taxonomy, ecology and geographic distribution of the genus Dunaliella Teod and prospects for its potential utilization. Naukova Dumka, Kiev, pp. 242.

[16] Azúa-Bustos A., González-Silva C., Salas L., Palma R. E. and Vicuña. 2010. A novel subaerial Dunaliella species growing on cave spiderwebs in the Atacama Desert. Extremophiles. 14 (5): 443-452.

[17] Preisig H. R. 1992. Morphology and taxonomy. In: Dunaliella: physiology, biochemistry, and biotechnology. Avron M. and A. Ben-Amotz (Eds). Boca Raton, CRC Press, pp. 1-15.

[18] Hejazi M. A., Barzegari A., Gharajeh N. H. and Hejazi M. S. 2010. Introduction of a novel 18S rDNA gene arrangement along with distinct ITS region in the saline water microalga Dunaliella. Saline Systems. 6: 4, http://dx.doi.org/10.1186/1746-1448-6-4.

[19] Zamani H., Moradshahi A. and Karbalaei-Heidari H. R. 2011 Characterization of a new Dunaliella salina strain MSI-1 based on nuclear rDNA ITS sequences and its physiological response to changes in composition of growth media. Hydrobiologia. 658 (1): 67-75.

[20] Li Z. and Xie S. L. 2006. Study on species and distribution of algae in salt lakes area in Yuncheng, Shanxi Province. J. Lake. Sci. 18 (2): 189-192.

[21] Sharma P., Agarwal V., Mohan M. K. Kachhwaha S. and Konthari S. L. 2012. Isolation and characterization of Dunaliella species from Sambhar Lake (India) and its phylogenetic position in the genus Dunaliella using $18 \mathrm{~S}$ rDNA. Natl. Acad. Sci. Lett. 35 (3): 207-213.

[22] Doyle J. J. 1987. A rapid DNA isolation procedure for small quantities of fresh leaf tissue. Phytochem. Bull. 19: 11-15.

[23] Olmos J., Paniagua J. and Contreras R. 2000. Molecular identification of Dunaliella sp. utilizing the18S rDNA gene. Lett. Appl. Microbiol. 30 (1): 80-84.

[24] Larkin M. A., Blackshields G., Brown N. P., Chenna R., McGettigan P. A., McWilliam H., Valentin F., Wallace I. M., Wilm A., Lopez R., Thompson J. D., Gibson T. J. and Higgins D. G. 2007. Clustal W and Clustal X version 2.0. Bioinformatics. 23 (21): 2947-2948.

[25] Tamura K., Peterson D., Peterson N., Stecher G., Nei M. and Kumar S. 2011. MEGA5: molecular evolutionary genetics analysis using maximum likelihood, 888 evolutionary distance, and maximum parsimony methods. Mol. Biol. Evol. 28 (10): 2731-2739.

[26] Guindon S. and Gascuel O. 2003. A simple, fast, and accurate algorithm to estimate large phylogenies by maximum likelihood. Systematic Biol. 52 (5): 696-704.

[27] Ronquist F. and Huelsenbeck J. P. 2003. MrBayes 3: bayesian phylogenetic inference under mixed models. Bioinformatics. 19 (12): 1572-1574.

[28] Posada D. 2008. jModel test: phylogenetic model averaging. Mol. Biol. Evol. 25: 1253-1256.

[29] Assunção P., Jaén-Molina R., Caujapé-Castells J., Jara A. D. L., Carmona L., Freijanes K. and Mendoza H. 2012. Phylogenetic position of Dunaliella acidophila (Chlorophyceae) based on ITS and rbcL sequences. J. Appl. Phycol. 24 (4): 635-639. 
[30] Hepperle D., Nozaki H., Hohenberger S. Huss V.A. Morita E. and Krienitz L. 1998. Phylogenetic position of the Phacotaceae within the Chlamydophyceaeas revealed by analysis of $18 \mathrm{~S}$ rDNA and rbcL sequences. J. Mol. Evol. 47: 420-430.
[31] Nakada T., Misawa K. and Nozaki H. 2008. Molecular systematics of Volvocales (Chlorophyceae, Chlorophyta) based on exhaustive 18S rRNA phylogenetic analyses. Mol. Phylogenet. Evol. 48 (1): 281-291. 